\title{
Operant behavior controlled by position of a moving object - a reinforcement learning model
} Cyril Brom*1, Daniel Klement ${ }^{2}$ and Michal Preuss ${ }^{1}$

Address: ${ }^{1}$ Dept. of Software and Computer Science Education, Faculty of Mathematics and Physics, Charles University in Prague, 11800 , Czech Republic and ${ }^{2}$ Dept. of Neurophysiology of Memory and Computational Neuroscience, Institute of Physiology, Academy of Sciences of the Czech Republic, Prague, 142 00, Czech Republic

Email: Cyril Brom* - brom@ksvi.mff.cuni.cz

* Corresponding author

from Seventeenth Annual Computational Neuroscience Meeting: CNS*2008

Portland, OR, USA. 19-24 July 2008

Published: II July 2008

BMC Neuroscience 2008, 9(Suppl I):P75 doi:10.1 186/147I-2202-9-SI-P75

This abstract is available from: http://www.biomedcentral.com/I47I-2202/9/SI/P75

(C) 2008 Brom et al; licensee BioMed Central Ltd.

\section{Description}

It has been demonstrated that operant behavior can be controlled by spatial stimuli. In one of our experiment, rats were conditioned to press a lever for reward when a moving object was passing through a particular region of the experimental room (unpublished data). Although the stimulus was changing smoothly, the transitions between rewarded and non-rewarded condition were sudden. Consequently the animals anticipated the arrival to the rewarded zone by responding in its vicinity.

We developed a reinforcement learning model to simulate this anticipatory behavior and to study its spatial and temporal components. An output neuron integrated inputs from four classes of sensory neurons: (1) neurons detecting the position of the object, (2) neurons indicating the time elapsed since the last reward and (3) since the last operant response, and (4) a neuron signaling the presence/absence of the reward. While the output neuron was a leaky-integrator with a binary activation function, a manner for sending a motor signal to press the lever, the sensory neurons were simple nodes lacking the time dynamic component that signaled the presence of a stimulus in their receptive field in a rate-coded manner. The synapses between the sensory neurons and the output neuron were modified according to a rule based on the Rescorla-Wagner rule [1]. The overall model resembles the spectral-timing model of Grossberg and Schmajuk [2] extended to the spatial domain.
Depending on the set up of learning parameters related to the different classes of sensory neurons, the network can learn the spatial and/or temporal features of the task resulting in spatial and/or temporal anticipation of the reward. The network well approximates data observed in real animals.

\section{Acknowledgements}

This work was supported by grants of MSMT (IM05 I7, LC554, and MSM0021620838) and research projects AVOZ50I I0509 and IETI003005I7.

\section{References}

I. Rescorla RA, Wagner AR: A theory of Pavlovian conditioning: The effectiveness of reinforcement and non-reinforcement. In Classical Conditioning II: Current Research and Theory New-York: Appleton-Century-Crofts; 1972:64-69.

2. Grosberg S, Schmajuk NA: Neural dynamics of adaptive timing and temporal discrimination during associative learning. Neural Networks 1989, 2:79-102. 